\title{
Double standards redux
}

\author{
RUTH MACKLIN
}

It is fair to say that nothing is more pressing in today's world than bringing an end to the Covid-19 pandemic. Or to be more precise, if not an end, then the quickest and most effective reduction of mortality and morbidity from the disease in every country where infections exist. The forthcoming theme issue of IJME explores a long-standing ethical concern in research with human beings, focused mainly on a question posed about vaccine research for prevention of Covid-19. Contributors from six continents address the question: In what circumstances - if any - is it ethically acceptable to use a research design in which the control group in a randomised, controlled trial (RCT) receives a placebo instead of a vaccine already approved for emergency use by regulatory bodies? That ethical question is not new. A controversy arose more than two decades ago in the context of research seeking a method of preventing maternal-to-child transmission of HIV/AIDS. At the heart of that controversy was a question about "double standards" in global research: Is it ethically permissible to conduct a type of research in low- and middle-income countries (LMICs) that would be impermissible in wealthier countries? (1) The question now rears its head again in the context of the Covid-19 pandemic.

An article published online in the Perspectives section of the New England Journal of Medicine on January 14 this year contends that new vaccine research using placebo controls should be carried out in countries that lack access to Covid-19 vaccines already approved for use in other countries. (2) The article says that "while it is still feasible and ethical," ongoing vaccine trials should use "directly randomized comparisons against placebo" to collect high-quality information. The purpose is "to obtain pivotal data to improve regulatory and public health decision making," including reliable information on longer-term safety and duration of protection (2).

The authors of the paper (hereinafter, WHO Ad Hoc Group) are several external experts convened by the World Health Organization (WHO), along with three WHO employees. WHO appointed the group to consult on next steps for Covid-19 vaccine evaluation, including what critical additional data should be sought. No one would quarrel with the need to obtain such data, since much is still unknown about features of the vaccines that have been given emergency approval.

\section{The historical context}

A crucial aspect of the debate over placebo controls in HIV prevention research in the 1990s was the context in which it arose. A medication highly effective for preventing foetal acquisition of AIDS had become available in resource-rich countries. But because of its cost, the method remained out of reach for virtually all low- and middle-income countries (LMICS) at the time. Pharmaceutical companies and the US National Institutes of Health (NIH) had begun to conduct placebo-controlled, mother-tochild HIV transmission studies of a new medication in Thailand, Uganda, and other LMICs. Critics argued that this embodied a "double standard" in the conduct of global research: one ethical rule for rich countries and another for poor countries (1). It would be unethical to withhold a proven prevention from pregnant women in a new clinical trial in the wealthier countries, when a successful method was already available. Defenders of the placebo-controlled design argued that this was the best way to obtain an affordable medication in the shortest possible time for the poor countries. Two such defenders at the time were the heads of the US government institutions that sponsored the placebo-controlled trials: the NIH and the Centers of Disease Control and Prevention (CDC) (3). These defenders did not contend that a trial using a proven medication for the control group could not obtain scientifically valid results. It is worth noting that a clinical trial using the same drug to prevent transmission of

Author: Ruth Macklin (macklinruth@gmail.com), Distinguished University Professor Emerita, Department of Epidemiology and Population Health, Albert Einstein College of Medicine, Bronx, NY 10461 USA.

To cite: Macklin R. Double standards redux. Indian J Med Ethics. 2021 Apr-Jun; 6(2) NS: 87-91. DOI: 10.20529/IJME.2021.021.

Published online first on March 19, 2021

(c) Indian Journal of Medical Ethics 2021 
HIV/AIDS from pregnant women to the foetus was carried out in Thailand without placebo controls. That study, also sponsored by the $\mathrm{NIH}$, was an equivalency trial comparing different regimens using the same drug (4)

\section{The WHO Ad Hoc Group's arguments}

According to the NEJM article, ongoing placebo-controlled vaccine trials for Covid-19 may still be occurring in high-income countries as well as in LMICs. However, a long-standing practice in research has called for either stopping ongoing trials when a successful product is available outside such trials, or at least informing trial participants that they may leave the study in order to access the newly available product. At the time of this writing, the following six vaccines are being distributed in different countries throughout the world: Pfizer-BioNTech, Moderna, Oxford/AstraZeneca, Johnson \& Johnson, Sputnik V, and Sinovac (5)

The bold claim made by the WHO Ad Hoc Group that such studies are "still ethical" is not explicitly defended in the article. Instead, the authors provide a rationale relying on a technical point. The stated rationale is that current vaccines are "still investigational... (under Emergency Use Listing...or similar regulatory mechanisms)."(2) This refers to the status of the vaccines authorised for use but not yet licensed by drug regulatory authorities in the US, the UK, the European Union, and other countries. Yet millions of doses of these vaccines are being deployed around the world, including in many middle-income countries and some low-income countries (Ghana is one example). Calling the vaccines "investigational" is technically correct since they have not been fully licensed. But it is misleading because ongoing data is not being collected from all of these millions of vaccinated individuals throughout the world. The article is proposing to do so for participants still in current placebo-controlled Phase 3 trials and those who will enrol in future Phase 3 vaccine trials. But that brings us back to the fundamental questions: Are future placebo-controlled Covid-19 vaccine trials ethically permissible even before such vaccines are fully licensed? As for participants in ongoing Phase 3 placebo-controlled trials, they should be informed that they may be eligible for a vaccine that has been approved under an emergency authorisation if they choose to leave the trial in which they are enrolled.

The WHO Ad Hoc Group contends that "a $70 \%$ effective single-dose vaccine may be more valuable than a two-dose regimen with $90 \%$ efficacy and greater implementation challenges. It is noteworthy that such a vaccine could not be identified without using placebo controls" (2). No methodological analysis is provided for why placebo controls are necessary, nor do the authors specify the respects in which the $70 \%$ effective vaccine is "more valuable."

It appears that speed is among the factors motivating the authors' preference for placebo controls. They acknowledge that "Randomized, noninferiority trials can provide clinically relevant data in some cases, but at a considerable cost to efficiency" (2). The article does not specify what the "some cases" are in which noninferiority trials can provide such data nor those cases in which such trials cannot. Efficiency includes lower costs as well as speed. While efficiency is an important value, it should not take precedence over ethics when the two conflict. With six vaccines already in the process of being deployed worldwide to millions of people, the current difficulties are largely those of implementation and logistics, as well as shortages of the products.

It is true that many unknowns continue to exist. An example is key information about the length of time for which the vaccines will remain effective. It is too soon after the emergency authorisations were approved to know how long vaccinated individuals remain protected. Another question is how effective the current vaccines are against the emerging variants of the virus that causes Covid-19. The US CDC cautions that early data show the vaccines may work against some variants, but could be less effective against others (6)

The key question is why placebo-controlled trials are needed to obtain such information. It is highly likely that new trials with this design would not be carried out in wealthy countries that already have access to the provisionally approved vaccines, even if the vaccine roll-out continues to be slow. And given the large number of middle-income countries that have begun to receive the vaccines manufactured in China and Russia, it is reasonable to question whether authorities in those countries would approve new placebo-controlled vaccine trials. That leaves most countries in Africa and a few in the Middle East and Central Asia that currently lack access to any of the six aforementioned vaccines.

This brings us to the WHO Ad Hoc Group's call for "firm commitments to maintaining blinded follow-up of participants in ongoing or future placebo-controlled trials until a licensed vaccine is fully deployed in the population." (2). That effectively means that participants cannot find out whether they were in the group that received the vaccines or in the placebo group, even after the study formally ends: "...we believe that trial sponsors are not ethically obligated to unblind treatment assignments for participants who desire to obtain a different investigational vaccine." (2) This provision effectively prevents past vaccine trial participants who received placebos from obtaining information that would enable them to protect themselves (and potentially other people) by getting one of the other conditionally approved vaccines.

What is involved in "firm commitments to maintaining blinded follow-up of participants" until a licensed vaccine is available in the population? This suggestion borders on a violation of the ethical requirement that research participants may terminate their 
participation at any time. Strictly speaking, it doesn't violate the rule because presumably, participants can leave the trial but still not be told whether they were in the placebo group or the group that received the vaccine. But in that case, what would be the point of not disclosing that information?

These questions prompt us to inquire about the informed consent process and documents the WHO Ad Hoc Group envisages for the trials it proposes. The many problems with research participants' understanding of consent forms have been documented in numerous empirical studies conducted over the years-too many to cite here. This situation is more complicated than usual in the context under discussion. The trial has ended, according to the authors, but participants are still being "followed." How is this to be explained in the informed consent process? If participants are being subjected to follow-up procedures, it seems likely they will believe they are still enrolled in the trial. In sum, to withhold from research participants in a Covid-19 vaccine RCT the information that they received a placebo, when others in their country or community are receiving vaccines authorised for emergency use (although not yet licensed) is nothing short of exploitation. In other words, the situation takes unfair advantage of former trial participants by deliberately leaving them worse off than others in their community or country.

The article notes that "participants in trials of such vaccines should have access to the standard of care in their location and, if the trial is successful, their communities should share in the benefit." This presumptive "standard of care" is better referred to as the "standard of prevention," a phrase used in an international ethics guidance document for HIV prevention research (7). In this situation, the "standard of prevention" could only mean a Covid-19 vaccine already in use in the country or community, whether or not it has already been licensed. This makes clear that the authors defend the use of placebo controls in a broader range of countries than the poorest ones currently lacking access to any Covid-19 vaccines. But we must also assume that people willing to enrol in a trial where they may be randomised to placebo are not yet eligible for the vaccine that exists in their country, that they are fully informed of the research design, and what that implies for their participation in the trial.

The WHO Ad Hoc Group says: "Countries with limited or no access to a known effective vaccine could thus ethically permit placebo-controlled trials of vaccines of potential relevance to them even if effective vaccines were already being marketed elsewhere." (2) How is this conclusion justified? Presumably, it refers to the requirement that the community would share in the benefit of a successful trial by gaining access to the resulting efficacious vaccine. But what if the research fails to yield an effective vaccine? Neither the experimental group nor the control group ends up with a preventive vaccine in such countries. The claim that all participants end up with the "standard of care" in such countries is a piece of sophistry. That phrase cannot meaningfully be used to refer to "no care" (in this case, "no prevention"). When people receive no therapeutic or preventive method, there can be no "standard' of care or prevention. The rationale for the conclusion is simply that no one in the vaccine trial is made worse off than before they entered the trial. This is a clear restatement of the ethically problematic view that "double-standards" in research are justifiable.

\section{International ethics guidance for research}

Authoritative sources provide guidance in controversies such as this. Two international documents are in widespread use throughout the world. The first of these is the Declaration of Helsinki (DoH), issued by the World Medical Association, which first appeared in 1964 and has undergone numerous revisions since then (8). The DoH does not have the status of international law. However, a number of countries cite the document as their official guidance for ethical review of proposed research, in addition to whatever domestic regulations they may have in place. For example, India has national guidelines that restrict the use of placebos when an effective option is available in the country (9).

Here are the relevant paragraphs in the current version of the DoH on the acceptability of placebo controls.

\section{Declaration of Helsinki (2013)}

Use of Placebo

33. The benefits, risks, burdens and effectiveness of a new intervention must be tested against those of the best proven intervention(s), except in the following circumstances:

Where no proven intervention exists, the use of placebo, or no intervention, is acceptable; or

Where for compelling and scientifically sound methodological reasons the use of any intervention less effective than the best proven one, the use of placebo, or no intervention is necessary to determine the efficacy or safety of an intervention and the patients who receive any intervention less effective than the best proven one, placebo, or no intervention will not be subject to additional risks of serious or irreversible harm as a result of not receiving the best proven intervention (8). 
As noted earlier, the article by the Ad Hoc Expert Group does not provide "compelling and scientifically sound methodological reasons" that the above paragraph in the DoH calls for. Nor is it the case that participants in proposed placebo-controlled vaccine studies "will not be subject to additional risks of serious or irreversible harm" if they are in the placebo group and acquire a serious case of Covid-19. Such cases may even be fatal.

The second authoritative source for international research ethics is a set of guidelines prepared by the Council for International Organizations of Medical Sciences (CIOMS), a non-governmental organisation based in Geneva, Switzerland (10). The relevant guideline is the following:

\section{CIOMS International Guidelines (2016)}

\section{Guideline 5. Choice of Control in Clinical Trials}

As a general rule, the research ethics committee must ensure that research participants in the control group of a trial of a diagnostic, therapeutic, or preventive intervention receive an established effective intervention.

Placebo may be used as a comparator when there is no established effective intervention for the condition under study, or when placebo is added on to an established effective intervention (10).

When there is an established effective intervention, placebo may be used as a comparator without providing the established effective intervention to participants only if:

- there are compelling scientific reasons for using placebo; and

- delaying or withholding the established effective intervention will result in no more than a minor increase above minimal risk to the participant and risks are minimized, including through the use of effective mitigation procedures. (10)

The second bullet point in CIOMS Guideline 5 specifies the level of risk that would be acceptable if the known intervention is withheld. In whatever way "a minor increase above minimal risk" is to be determined, it is clear that the intent is to avoid serious risks such as those of Covid-19.

These two international ethics guidance documents clearly reject the acceptability of placebo-controlled trials in circumstances like the current pandemic. It is important to note in this context that the CIOMS publication cites WHO as a collaborator in drafting its guidelines. The cover and title page say:"Prepared by the Council for International Organizations of Medical sciences (CIOMS) in collaboration with the World Health Organization (WHO)." The Acknowledgements page in the document says: "As a result of this collaboration, the guideline development process is consistent with the standards and policies of WHO" (10: p. iii). The acknowledgement further notes that the guidelines received "organization-wide review by WHO especially by the Ethics Review Committee...." (10: p.iii). Furthermore, the WHO website contains this statement regarding the governance of its Ethics Review Committee (ERC): "The ERC is guided in its work by the World Medical Association Declaration of Helsinki (1964) last updated in 2013 as well as the International Ethical Guidelines for Biomedical Research Involving Human Subjects (CIOMS 2016)."(11)

It is evident from these acknowledgments that the World Health Organization has an obligation to adhere to the spirit and letter of the DoH and the CIOMS guidelines. The article published in NEJM, under the authorship of a WHO Ad Hoc Group, violates the World Health Organization's own stated ethical commitment to adhere to these two international guidelines for research with human beings.

\section{References}

1. Lurie P, Wolfe SM. Unethical trials of interventions to reduce perinatal transmission of the Human Immunodeficiency Virus in Developing Countries. NEngl J Med. 1997 Sep 18; 337(12):853-6.

2. WHO Ad Hoc Expert Group on the Next Steps for Covid-19 Vaccine Evaluation, Placebo-Controlled Trials of Covid-19 Vaccines - Why We Still Need Them. N Engl J Med. 2021 Jan 14; 384(2): e2(1)-e2(3). Epub 2020 Dec 2.

3. Varmus H, Satcher D. Ethical Complexities of Conducting Research in Developing Countries. N Engl J Med. 1997 Oct 2; 337(14):1003-5.

4. Lallemant M, Jourdain G, Le Coeur S, Kim S, Koetsawang S, Comeau AM et al. A trial of shortened zidovudine regimens to prevent mother-to-child transmission of human immunodeficiency virus Type 1. Perinatal HIV Prevention Trial (Thailand) Investigators. N Engl J Med. 2000 Oct 5; 343(14):98291. Doi: 10.1056/NEJM200010053431401.

5. Lawler D. Latin America turns to China and Russia for COVID-19 vaccines. Axiox, 2021 Mar 2[cited 2021 Mar 2]. Available from: https:// www.axios.com/russia-chinese-vaccines-latin-america-us-mexico-86a9daf5-4d39-421b-958a-40a457513e1c.html

6. Centers for Disease Control and Prevention. When You've Been Fully Vaccinated: How to Protect Yourself and Others. Atlanta, GA: CDC; 2021 Mar 9 [cited 2021 Mar11]. Available from: https://www.cdc.gov/coronavirus/2019-ncov/vaccines/fully-vaccinated.html

7. UN AIDS and WHO. Ethical Considerations in HIV Prevention Trials. 2021[cited 2021 Mar 2]. Available from: https://www.unaids.org/sites/default/files/ media_asset/ethical-considerations-hiv-prevention-trials_en.pdf.

8. World Medical Association, WMA Declaration of Helsinki-Ethical Principles for Medical Research Involving Human Subjects. 2013 Oct [cited 2021 Mar 2]. Available from: https://www.wma.net/policies-post/wma-declaration-of-helsinki-ethical-principles-for-medical-research-involving-humansubjects/

9. Indian Council of Medical Research. National Ethical Guidelines for Biomedical and Health Research Involving Human Participants. New Delhi: ICMR; 
2017[cited 2021 Mar 2]. Available from:https://main.icmr.nic.in/sites/default/files/guidelines/ICMR_Ethical_Guidelines_2017.pdf

10. Council for International Organisations of Medical Sciences (CIOMS) and World Health Organization (WHO). International Ethical Guidelines for Health-related Research Involving Humans. Geneva: CIOMS and WHO; 2016[cited 2021 Mar 2]. Available from: https://cioms.ch/wp-content/uploads/ 2017/01/WEB-CIOMS-EthicalGuidelines.pdf

11. World Health Organization. Research Ethics Review Committee (REC). About us. Geneva: WHO; date unknown [cited 2021 Mar 2]. Available from: https://www.who.int/groups/research-ethics-review-committee/about

\section{Thank You, Reviewers!}

We are grateful to our reviewers for the dedicated work they put into evaluating and improving submissions. Besides our core group of Editors, we thank the editorial board members and others who reviewed manuscripts during the year 202021:

Aamir Jafarey, Aarthy Ramasamy, Aarthi Raveendiran, Aasim Ahmad, Abha Saxena, Abida Sultana, Akhila Vasan, Alastair McLennan, Alex London, Alok Sarin, Anindita Majumdar, Anjali Nair, Arun Bhatt, Arun Kumar Annamalai, Astrid Lobo Gajiwala, Bernard Dickens, Bevin Vijayan, Bijoya Roy, Bushra Shirazi, Catherine Slack, Charles Weijer, Deepa Pandit, Deepa Venkatachalam, Denny John, Dheeraj Kattula, Dhvani Mehta, Dipika Jain, Eldred Tellis, Eric Meslin, Florencia Luna, George Thomas, Hareesh Angothu, Ido Solt, Ignacio Mastroleo, Ishita Goyal, Jagriti Gangopadhyay, Jasodhara Dasgupta, Jayanta Bhattacharya, Jing-Bao Nie, J Devika, Jissa VT, JK Lakshmi, Joe Varghese, Kenneth Zysk, Ketaki Chowkhani, KS Jacob, Lakshmi Lingam, Lars Breimer, Lopa Mehta, Md Abid Geelani, Madhurima Nundy, Malu Mohan, Manickam Ponnaiah, Manjulika Vaz, Mario Vaz, Monica Sakhrani, Monty Khajanchi, Nagendra Dudi-Venkata, Nandini Kumar, Naveen Kumar, Nithya Gogtay, Nobhojit Roy, Omesh Bharti, Padmaja Shaw, Pragati Hebbar, Pragna Rao, Pravin Bolshete, Priya Satalkar, Priyadarshini C, Priya Pais, Radha D'Souza, Radha Holla Bhar, Raj Kumar Mani, Rakhal Gaitonde, Rakesh PS, Raman Kutty, Ramesh Awasthi, Ravi Prasad Varma, Ravi Vaswani, Reike van der Graaf, Richard A Cash, Rubesh Kumar, Ruth Macklin, Saima lqbal, Sadhana Natu, Samiran Nundy, Sandeep Bawdekar, Santosh Vijayakumar, Satendra Singh, Saumil Dholakia, Saurav Basu, Sayori Ghoshal, Shahista Parveen, Shaibya Saldanha, Shilpa Phadke, Shining Star Lyngdoh, Shireen Jeejeebhoy, Shrinidhi Datar, Siddharth Sarkar, Smitha Deshpande, Sophia Modi, Soumitra Pathare, Subhasri B, Suchitra Dalvie, Sudarshini Subramaniam, Sudarshan Kottai, Sudha Ramalingam, Sujit Chandy, Sundar Sarukkai, Sunil K Pandya, Sunil Rajpal, Sunu C Thomas, Supriya Subramani, Suthanthira Kannan, Swarnalakshmi Singaravelu, Sylvia Karpagam, Upreet Dhaliwal, Usha Raman, Vallish BN, Varalakshmi Elango, Vipin Vashishtha, William Joe, Yogesh Jain, 